\title{
IRREDUCIBLE REPRESENTATIONS OF LIE ALGEBRA EXTENSIONS
}

\author{
BY RICHARD E. BLOCK ${ }^{1}$
}

\section{Communicated by Alex Rosenberg, December 30, 1973}

This note announces three density theorems involving representations of Lie algebras and associative algebras. The first theorem describes the irreducible (possibly infinite dimensional) representations $\rho$ of a Lie algebra $\mathfrak{g}$ with an ideal $\mathfrak{f}$ such that the restriction of $\rho$ to $\mathfrak{f}$ has some absolutely irreducible quotient representation. The second result is an embedding theorem for the irreducible representations of the Weyl algebras $A_{n, C}$ over $C\left(A_{n, C} \cong C\left[t_{1}, \cdots, t_{n}, \partial / \partial t_{1}, \cdots, \partial / \partial t_{n}\right]\right.$, the associative algebra of partial differential operators on $n$ variables with coefficients in the polynomial ring $\left.C\left[t_{1}, \cdots, t_{n}\right]\right)$. Our result is a sort of algebraic analogue of the uniqueness of the Heisenberg commutation relations, and has an application to irreducible representations of nilpotent Lie algebras via Dixmier's theory [5]. The third theorem describes the differentiably simple algebras having a maximal ideal. This result unifies the author's theorem [3] on differentiably simple rings with a minimal ideal, and Guillemin's theorem [7], [2] on the structure of a nonabelian minimal closed ideal of a linearly compact Lie algebra.

1. In what follows, all algebras, tensor products etc., will be over an arbitrary given field $\Phi$, unless otherwise stated. If the characteristic is prime, the Lie algebras considered will always be assumed restricted (=Lie $p$-algebra), and the same for their homomorphisms, ideals, etc. Also $U$ will denote the universal enveloping algebra functor at characteristic 0 , and the restricted universal enveloping algebra functor at prime characteristic. We shall take $\mathfrak{g}$ to be a given Lie algebra, and $\mathfrak{f}$ an ideal of g.

Recall that if $V$ is a $\mathfrak{f}$-module with corresponding representation $\sigma$, then the stabilizer $\operatorname{St}(V, \mathfrak{g})$ of $V$ in $\mathfrak{g}$ is defined [1], [6] by

$$
\operatorname{St}(V, \mathfrak{g})=\{x \in \mathfrak{g} \mid \exists \eta \in \operatorname{Hom}(V, V) \ni \sigma[x, y]=[\eta, \sigma y] \forall y \in \mathfrak{t}\} .
$$

This is a subalgebra of $\mathfrak{g}$ containing $\mathfrak{f}$, and gives the analogue of the concept of stabilizer for group representations.

AMS (MOS) subject classifications (1970). Primary 16A64, 17B10, 17A99; Secondary 17B30, 17B65, 16A72.

${ }^{1}$ This research was partially supported by NSF Grant GP-23998. 
At characteristic 0 , Blattner [1] has shown that if $V$ is a $\mathfrak{f}$-module which is absolutely irreducible (i.e. $V$ is irreducible and has centralizer $\Phi$ ), if $\mathfrak{h}=\operatorname{St}(V, \mathfrak{g})$, and if $W$ is an irreducible $\mathfrak{h}$-module which as $\mathfrak{f}$-module is a direct sum of copies of $V$, then the induced $\mathfrak{g}$ module $U \mathfrak{g} \otimes_{U \mathfrak{h}} W$ is irreducible; Dixmier [6] has used this result to show that every irreducible $\mathfrak{g}$-module containing an absolutely irreducible $\mathfrak{f}$-submodule is isomorphic to such an induced module. It can be shown that the Blattner-Dixmier theorem remains valid in the (restricted) prime characteristic case.

We now turn to the more complicated situation of coinduced modules and irreducible $\mathfrak{g}$-modules with a maximal $\mathfrak{f}$-submodule. In topological considerations $\mathfrak{g}$ will have the discrete topology unless otherwise stated. If $\mathfrak{h}$ is a subalgebra of $\mathfrak{g}$ and $W$ is an $\mathfrak{h}$-module then, regarding $U \mathfrak{g}$ as a $U \mathfrak{h}, U \mathfrak{g}$-bimodule, we get the coinduced $U \mathfrak{g}$-module $\operatorname{Hom}_{U \mathfrak{h}}(U \mathfrak{g}, W)$. (This gives the right adjoint to the forgetful functor from $U \mathfrak{g}$-modules to $U \mathfrak{h}$-modules; the left adjoint is given by the induced $U \mathfrak{g}$-module $U \mathfrak{g} \otimes_{U \mathfrak{h}} W$.) If $W$ is a topological $U \mathfrak{h}$-module we give $\operatorname{Hom}_{U \mathfrak{h}}(U \mathfrak{g}, W)$ the finite-open topology.

LEMma 1. Let $V$ be an absolutely irreducible $\mathfrak{f}$-module, ha a subalgebra of $\mathrm{g}$ containing $\operatorname{St}(V, \mathfrak{g})$, and $W$ a topological $\mathfrak{h}$-module with a family $\left\{\pi_{\imath}\right\}_{i \in I}$ of $\mathfrak{f}$-maps $\pi_{i}: W \rightarrow V$ such that the topology on $W$ is that induced by $\left\{\pi_{i}\right\}_{i \in I}$ (i.e. the weakest topology making all $\pi_{i}$ continuous) where $V$ is discrete. Then the coinduced $U_{\mathfrak{g}-\text {-module, }} \operatorname{Hom}_{U \mathfrak{h}}(U \mathfrak{g}, W)$, is topologically irreducible if (and only if) $W$ is.

Blattner [1], [2] has proved a related result for the case in which $V$ is linearly compact and topologically absolutely irreducible and $W$ (as $\mathfrak{f}$-module) is a product of copies of $V$.

Note that $\operatorname{Hom}_{U \mathfrak{b}}(U \mathfrak{g}, W)$ above has a maximal $\mathfrak{f}$-submodule. The following seems to be the first result in the converse direction, i.e. describing the irreducible $\mathfrak{g}$-modules having a maximal $\mathfrak{f}$-submodule.

THEOREM 1. Let $M$ be an irreducible g-module having a (maximal) $\mathfrak{f}$-submodule $N$ such that the quotient $V=M \mid N$ is absolutely irreducible (as a $\mathfrak{\mathfrak { f }}$-module); give $V$ the discrete topology, and let $\mathfrak{h}=\operatorname{St}(V, \mathfrak{g})$. Then there is a topological h-module $W$ which as a $\mathfrak{\mathfrak { f }}$-module is a dense topological submodule of a product of copies of $V$ such that $M$ is isomorphic to a dense submodule of the coinduced module $\operatorname{Hom}_{U \mathfrak{\natural}}(U \mathfrak{g}, W)$.

Here $W$ can be taken to be the quotient of $M$ modulo the largest $\mathfrak{h}$-submodule contained in $N$. It follows from the theorem that the annihilator (in $U \mathfrak{g}$ ) of $M$ equals the largest (two-sided) ideal of $U \mathfrak{g}$ contained in $P(U \mathfrak{g})$ where $P$ is the annihilator (in $U \mathfrak{h}$ ) of $W$. By Quillen's lemma [8], the hypothesis that the irreducible module $V$ be absolutely irreducible can be deleted if $\mathfrak{f}$ is finite dimensional and $\Phi$ is algebraically closed. 
For brevity, the results above have been stated in less than their full generality. We remark in particular that they have a very useful extension to analogous results where one is given an associative algebra $B$ (with 1 ) in place of $\mathfrak{E}$, and an action of $\mathfrak{g}$ on $B$ by derivations (i.e. a homomorphism $\zeta$ of $g$ to Der $B$ ). Then one can form the smash (or semidirect) product $B \# U \mathfrak{g}$ (see [9]). Analogues of Lemma 1 and Theorem 1 (with $V$ a $B$ module, $W$ a $B \# U h$-module, and $M$ a $B \# U \mathfrak{g}$-module) can be shown for the coinduced $B \# U \mathfrak{g}$-module $\operatorname{Hom}_{B \# U \mathfrak{h}}(B \# U \mathfrak{g}, W)$ which in fact is $U \mathfrak{g}$-module isomorphic (and homeomorphic) to $\operatorname{Hom}_{U \mathfrak{h}}(U \mathfrak{g}, W)$. Similarly we can extend the Blattner-Dixmier theorem to a result on the induced $B \# U \mathfrak{g}$-module $B \# U \mathfrak{g} \otimes_{B \# U \mathfrak{h}} W$ (which is $U \mathfrak{g}$-module isomorphic to $\left.U \mathfrak{g} \otimes_{U \mathfrak{h}} W\right)$. These results on $B \# U \mathfrak{g}$-modules can be applied in the study of differentiably irreducible modules [4] and are used in the proofs of Theorems 2 and 3 below.

2. The Weyl algebra $A_{n}(n \geqq 0)$ over $\Phi$ is the associative algebra with unit with generators $x_{1}, \cdots, x_{n}, y_{1}, \cdots, y_{n}$ subject to the relations

$$
\left[x_{i}, x_{j}\right]=\left[y_{i}, y_{j}\right]=0, \quad\left[x_{i}, y_{j}\right]=\delta_{i j} \quad(i, j=1, \cdots, n) .
$$

A faithful representation $\rho$ of $A_{n}$ in $\Phi\left[\left[t_{1}, \cdots, t_{n}\right]\right]$ (formal power series in $n$ indeterminates) is given via $\rho\left(x_{i}\right)=\partial / \partial t_{i}, \rho\left(y_{i}\right)=\mu\left(t_{i}\right)$, where $\mu\left(t_{i}\right)$ denotes the multiplication by $t_{i}$. Then $\Phi\left[t_{1}, \cdots, t_{n}\right]$ is an irreducible $A_{n}$-submodule.

In order to state our result on the irreducible representations of $A_{n}$, we define a special automorphism $\theta$ of $A_{n}$ to be an automorphism such that for $i=1, \cdots, n$ and some scalars $\alpha_{1}, \cdots, \alpha_{n}$, either $\theta x_{i}=x_{i}$ and $\theta y_{i}=y_{i}-\alpha_{i} 1$ or $\theta x_{i}=-y_{i}$ and $\theta y_{i}=x_{i}-\alpha_{i} 1$. Given such a $\theta, \rho \theta$ is a representation of $A_{n}$ in $\Phi\left[\left[t_{1}, \cdots, t_{n}\right]\right]$, i.e. we get an $A_{n}$-module, $\Phi\left[\left[t_{1}, \cdots, t_{n}\right]\right]_{\theta}$, where for each $i$ either $x_{i}$ acts by $\partial / \partial t_{i}$ and $y_{i}$ by $\mu\left(t_{i}\right)-$ $\alpha_{i} 1$, or $y_{i}$ acts by $-\partial / \partial t_{i}$ and $x_{i}$ by $\mu\left(t_{i}\right)-\alpha_{i} 1$.

THEOREM 2. Let $\Phi$ be an algebraically closed nondenumerable field of characteristic $0, A_{n}$ the Weyl algebra over $\Phi$ (with generators $x_{1}, \cdots, x_{n}$, $y_{1}, \cdots, y_{n}$ as above), and $M$ an irreducible $A_{n}$-module. Then $M$ is isomorphic to a submodule of $\Phi\left[\left[t_{1}, \cdots, t_{n}\right]\right]_{\theta}$ for some special automorphism $\theta$.

Every nonzero submodule of $\Phi\left[\left[t_{1}, \cdots, t_{n}\right]\right]_{\theta}$ is dense in $\Phi\left[\left[t_{1}, \cdots, t_{n}\right]\right]$. The polynomials form an irreducible submodule of $\Phi\left[\left[t_{1}, \cdots, t_{n}\right]\right]_{\theta}$, but an abundance of others $(|\Phi|$ nonisomorphic ones) can be exhibited.

Combining Theorem 2 with a theorem of Dixmier [5] (whose work on the irreducible representations of nilpotent finite-dimensional Lie algebras in a sense reduces their classification to that of the irreducible representations of $A_{n}$ ), we get the following. 
COROLlaRY 1. With $\Phi$ as in Theorem 2 , let $\tau$ be an irreducible representation of a finite-dimensional nilpotent Lie algebra $\mathfrak{g}$. Then for some $n \geqq 0$, $\tau$ is equivalent to a subrepresentation of a representation $\psi$ of $\mathfrak{g}$ acting in $\Phi\left[\left[t_{1}, \cdots, t_{n}\right]\right]$ such that the sets $\{\psi(x) \mid x \in \mathfrak{g}\}$ and $\left\{\partial / \partial t_{2}, \mu\left(t_{i}\right) \mid i=1, \cdots, n\right\}$ generate the same subalgebra of endomorphisms of $\Phi\left[\left[t_{1}, \cdots, t_{n}\right]\right]$.

3. The next theorem determines the structure of certain algebras with no proper ideal invariant under a given family of derivations. Before stating it we need some preliminary notions. Suppose $S$ is a (not necessarily associative) simple algebra. The centroid (or multiplication centralizer) $\Gamma$ of $S$ is the subalgebra of elements of $\operatorname{Hom}_{\Phi}(S, S)$ which commute with all the left and right multiplications of $S$. Since $S$ is simple, $\Gamma$ is a field, and $S$ is also an algebra over $\Gamma$. The scalar extension of the given Lie algebra $g$ to $\Gamma$ will be denoted by $\mathfrak{g}_{\Gamma}$. Since $U \mathfrak{g}_{\Gamma}$ is a coalgebra, $\operatorname{Hom}_{\Gamma}\left(U \mathfrak{g}_{\Gamma}, S\right)$ is an algebra under the convolution multiplication, and $\operatorname{Hom}_{\Gamma}\left(U \mathfrak{g}_{\Gamma}, S\right)$ satisfies any multilinear identities that $S$ does. If $\mathfrak{h}$ is a subalgebra of $\mathfrak{g}_{\Gamma}$ and $\mathfrak{h}$ acts on $S$ (as an algebra over $\Gamma$ ) by derivations, then it may easily be shown that $\operatorname{Hom}_{U_{\mathfrak{h}}}\left(U \mathfrak{g}_{\Gamma}, S\right)$ is a subalgebra of $\operatorname{Hom}_{\Gamma}\left(U \mathfrak{g}_{\Gamma}, S\right)$. By a topological $g$-algebra we shall mean a topological algebra on which $\mathfrak{g}$ acts by continuous derivations. If $S$ is a topological algebra then $\operatorname{Hom}_{\Gamma}\left(U \mathfrak{g}_{\Gamma}, S\right)$ is a topological g-algebra. A topological $\mathfrak{g}$-algebra will be called topologically $\mathfrak{g}$-simple if it has no proper closed ideal invariant under $\mathrm{g}$.

THEOREM 3. Let $R$ be a topological g-algebra (possibly nonassociative or discrete) which is topologically $\mathrm{g}$-simple. Suppose $R$ has a closed maximal ideal $N$, write $\Gamma$ for the centroid of the (simple) algebra $S=R / N$, and assume that each $\gamma$ in $\Gamma$ is continuous (on $S$ ) and that $\Gamma$ is separable algebraic over $\Phi$. Then there is a subalgebra $\mathfrak{h}$ of $\mathfrak{g}_{\Gamma}$ and a continuous isomorphism (of $\mathfrak{g}$-algebras) of $R$ onto a dense $\mathfrak{g}$-subalgebra of $\operatorname{Hom}_{U \mathfrak{b}}\left(U_{\mathfrak{g}_{\Gamma}}, S\right)$.

It can easily be seen that $\operatorname{Hom}_{U \mathfrak{h}}\left(U \mathfrak{g}_{\Gamma}, S\right)$ is isomorphic as a topological algebra to $S\left[\left[X_{j}\right]\right]_{j \in J}$, the formal power series in the indeterminates $\left\{X_{j}\right\}_{j \in J}$ with coefficients in $S$, where the indeterminates are $p$-truncated $\left(X_{j}^{p}=0\right.$ for all $j$ ) in case the characteristic is a prime $p$. Here the cardinality of $J$ equals the codimension of $\mathfrak{h}$ in $\mathfrak{g}_{\Gamma}$.

We mention two important special cases as applications of Theorem 3 . First, suppose that $R$ is a discrete (not necessarily associative) algebra. Recall that $R$ is called differentiably simple if $R^{2} \neq 0$ and if there is a set $D$ of derivations of $R$ (one might as well take $D=\operatorname{Der} R$ ) such that $R$ has no proper $D$-ideal, i.e. ideal invariant under $D$. For example, a nonabelian minimal ideal of a Lie algebra is differentiably simple. The main result of [3] says that if $R$ is differentiably simple and has a minimal (two-sided) 
ideal then $R$ has a (unique) maximal ideal $N$ and either $R$ is simple (i.e. $R=R / N$ ) or $\Phi$ has prime characteristic $p$ and $R$ is isomorphic to the algebra of $p$-truncated polynomials in some finite set of indeterminates with coefficients in the simple algebra $R / N$. The special case when $R$ is finite dimensional and $\Phi$ is perfect is thus generalized by Theorem 3 (with $\mathrm{g}=\operatorname{Der} R$ ).

The second application concerns topological Lie algebras which are linearly compact. Guillemin [7] has proved that if $R$ is a nonabelian minimal closed ideal of such a Lie algebra then $R$ has a (unique) closed maximal ideal $N$, the centroid $\Gamma$ of the simple Lie algebra $R / N$ is a finitedimensional extension of $\Phi$, and if, in addition, $\Phi$ has characteristic 0 , then $R$ is isomorphic to the algebra $(R / N)\left[\left[X_{j}\right]\right]_{j \in J}$ of formal power series for some finite set $J$. In our case we can obtain Guillemin's theorem (and a little more) as a corollary of Theorem 3 ; moreover the result remains valid at prime characteristic (with the $X_{j} p$-truncated).

Blattner [2] has given another proof of Guillemin's theorem when $\Phi$ is algebraically closed (actually when $\Gamma=\Phi$ ) at characteristic 0 . Our proof to an extent resembles Blattner's.

\section{REFERENCES}

1. R. J. Blattner, Induced and produced representations of Lie algebras, Trans. Amer. Math. Soc. 144 (1969), 457-474. MR 46 \#7338a.

2. - A theorem of Cartan and Guillemin, J. Differential Geometry 5 (1970), 295-305. MR 46 \#7338b.

3. R. E. Block, Determination of the differentiably simple rings with a minimal ideal, Ann. of Math. (2) 90 (1969), 433-459. MR 40 \#4319.

4. —_, Modules over differential polynomial rings, Bull. Amer. Math. Soc. 79 (1973), 729-733.

5. J. Dixmier, Représentations irréductibles des algèbres de Lie nilpotentes, An. Acad. Brasil. Ci. 35 (1963), 491-519. MR 32 \#165.

6. - Sur les représentations induites des algebres de Lie, J. Math. Pures Appl. 50 (1971), 1-24.

7. V. Guillemin, A Jordan-Hölder decomposition for a certain class of infinite dimensional Lie algebras, J. Differential Geometry 2 (1968), 313-345. MR 41 \#8481.

8. D. Quillen, On the endomorphism ring of a simple module over an enveloping algebra, Proc. Amer. Math. Soc. 21 (1969), 171-172. MR 39 \#252.

9. M. E. Sweedler, Hopf algebras, Mathematics Lecture Notes Series, Benjamin, New York, 1969. MR 40 \#5705.

Department of Mathematics, University of California, Riverside, California 92502 